\title{
Editorial on special topic: sirtuins in metabolism, aging, and disease
}

\author{
Aleksey G. Kazantsev ${ }^{1 *}$ and Tiago F. Outeiro ${ }^{2}$ \\ Department of Neurology, Harvard Medical School and Massachusetts General Hospital, Boston, MA, USA \\ 2 Department of NeuroDegeneration and Restorative Research, University Medizin Goettingen, Goettingen, Germany \\ *Correspondence: akazantsev@partners.org
}

The sirtuin family of $\mathrm{NAD}^{+}$-dependent enzymes has received much of attention in recent years due to their diverse physiological functions in metabolism, aging, and age-related human diseases. The mammalian sirtuins (SIRT1-7) act as $\mathrm{NAD}^{+}$-dependent protein deacetylases and weak mono-ADP-ribosyl transferases on a variety of targets, including histones, transcription factors, and apoptotic modulators. The sirtuins appear to be the key sensors for available energy stores, which function as molecular switch between protein acetylation and metabolism. Furthermore, it has been shown in a broad range of experimental disease models, from yeast to mouse models, that modulation of sirtuin activities, particularly that of the most studied SIRT1 protein, suppresses or ameliorates pathological states, and thus sirtuins constitute attractive novel therapeutic targets for many age-related disorders, for most metabolic disorders such as diabetes and obesity.

The major goal of this Special Issue is to discuss and evaluate the current knowledge on sirtuin biology for a rational approach toward the development sirtuin-based therapeutics.

In the article "Sirtuins as regulators of the yeast metabolic network" the authors discuss a network complexity of yeast SIRT1 homolog. The archetypal sirtuin, yeast silent information regulator2 (SIR2), is an $\mathrm{NAD}^{+}$dependent protein deacetylase that interacts with metabolic enzymes glyceraldehyde-3-phosphate dehydrogenase and alcohol dehydrogenase, as well as enzymes involved in $\mathrm{NAD}(\mathrm{H})$ synthesis, that provide or deprive $\mathrm{NAD}^{+}$in its close proximity. This influences sirtuin activity, and facilitates a dynamic response of the metabolic network to changes in metabolism with effects on physiology and aging. The molecular network downstream Sir2, however, is complex, and tertiary interactions of Sir2, relevant to metabolic regulation, involve half of the yeast proteome.

The SIRT1 network is further discussed in the manuscript "Interactomic and pharmacological insights on human SIRT1." The article presents studies on human SIRT1, which illuminate functional relationships of gene-protein interactions, controlling major metabolic pathways. This article also shows the mechanism of binding of small molecule activators and discusses critical structural determinants and physico-chemical features important for rational design of SIRT1 effectors.

Rational drug design is also the topic of the article "Structures, substrates, and regulators of mammalian Sirtuins - opportunities and challenges for drug development." The authors recapitulate advances in structural and mechanistic studies on substrate recognition and deacetylation by sirtuins, which provides a basis for the identification and characterization of small molecule sirtuin activators and inhibitors as well as evaluation the molecular mechanisms regulating their activities.
The importance of posttranslational modifications for sirtuin activities is discussed in the article "Regulation of sirtuin function by posttranslational modifications." Here the authors review the posttranslational regulation mechanisms of mammalian sirtuins and discuss their relevance regarding the physiological processes. Based on available data they suggest that the $\mathrm{N}$ - and $\mathrm{C}$-termini are the targets of posttranslational modifications, which in turn determines differential interaction with catalytic core domain. The authors emphasize the key regulatory roles of the $\mathrm{N}$ - and C-termini in controlling different signaling pathways.

The article "Role and therapeutic potential of the pro-longevity factor FOXO and its regulators in neurodegenerative disease" is focused on downstream sirtuin targets, critical for aging, and age-related neurodegeneration. The author discusses FOXO network of transcription factors, which is most notable for its association with longevity and modulation by upstream regulators such as sirtuins. He emphasizes that the FOXO network is a complex stress response system and thus development of diseasemodifying strategies requires careful examination. C. Neri argues that although the FOXO network contains druggable genes such as sirtuins, whether they should be activated or inhibited and whether protection against the early or late phases of neuronal cell decline might require opposite therapeutic strategies remains unclear.

Next, the complexity of therapeutic targeting of sirtuins is discussed in the article "SIRT2 as a therapeutic target for age-related disorders." The authors discuss the second member of sirtuin family, SIRT2, its physiological role(s) in various cellular environments and tissue-specific functions. This article summarizes the main scientific advances on SIRT2 protein biology and explores its potential as a therapeutic target for treatment of age-related disorders.

In the article "Inhibition of sirtuin 2 with sulfobenzoic acid derivative AK1 is non-toxic and potentially neuroprotective in a mouse model of frontotemporal dementia" the authors provide experimental evidence on the efficacy of SIRT2 inhibitor in mouse brain. The rTg4510 mouse model, which expresses a mutant form of the tau protein associated with FTD with Parkinsonism, undergoes dramatic hippocampal and cortical neuronal loss. In present study, the SIRT2 inhibitor was directly delivered to the hippocampus with an osmotic minipump. The treatment was found to be safe in wild-type and transgenic mice and, furthermore, it provided neuroprotection in the $\mathrm{rTg} 4510$ hippocampal circuitry. This study provides proof-of-concept for therapeutic benefits of SIRT2 inhibitors in both tau-associated FTD and Alzheimer's disease. 
An apparent link between sirtuin modalities and cancer has emerged and is discussed in the two articles, "Sirtuins and disease: the road ahead" and "Sirtuins, Metabolism, and Cancer." In the first article the authors discuss experimental evidences suggesting a critical role for SIRT1 in tumor initiation and progression as well as drug resistance by blocking senescence and apoptosis, and by promoting cell growth and angiogenesis. The present article highlights sirtuin mechanism(s) of action and deregulation in cancer, and focuses on the therapeutic potential of sirtuin modulators both in cancer prevention and treatment. In the second paper authors specifically review recent progress on the role of sirtuins in DNA repair and energy metabolism, and further address the sirtuin role(s) in the biology of cancer.
Overall, the present special issue "Sirtuins in Biology and Disease" provides an overview and insight on therapeutic potentials of sirtuin-based therapeutics and discusses the evident complexity of drug-targeting these modalities for human indications.

Received: 04 April 2012; accepted: 05 April 2012; published online: 24 April 2012.

Citation: Kazantsev AG and Outeiro TF (2012) Editorial on special topic: sirtuins in metabolism, aging, and disease. Front. Pharmacol. 3:71. doi: 10.3389/fphar.2012.00071 This article was submitted to Frontiers in Experimental Pharmacology and Drug Discovery, a specialty of Frontiers in Pharmacology.

Copyright $\odot 2012$ Kazantsev and Outeiro. This is an open-access article distributed under the terms of the Creative Commons Attribution Non Commercial License, which permits non-commercial use, distribution, and reproduction in other forums, provided the original authors and source are credited. 